\title{
Adaptación y Propiedades Psicométricas de la Escala de Dependencia y Adicción al Smartphone "EDAS" para medir conductas adictivas al Smartphone en adolescentes y jóvenes de 18 a 35 años de la ciudad de Puno - 2020
}

\begin{abstract}
Adaptation and Psychometric Properties of the Smartphone Dependence and Addiction Scale "EDAS" to measure addictive behaviors to the Smartphone in adolescents and young people between 18 and 35 years of the city of Puno - 2020
\end{abstract}

\author{
Aurora Lucana Hancco' ${ }^{1}$, Heber Pari Betancur², Alcides Quispe Mamani ${ }^{3}$
}

\section{RESUMEN}

Objetivo: Adaptar y analizar las propiedades psicométricas de la escala de "Dependencia y Adicción al Smartphone" (EDAS) autoría original de María Aranda López, Virginia Fuentes Gutiérrez y Marta García Domingo, quienes crearon el instrumento el año 2017. Metodología: Se tuvo una muestra de 117 personas de edades de 18 a 35 años entre varones y mujeres, la recolección de datos se hizo vía virtual por la plataforma de Google formulario, los resultados demuestran que la escala de Dependencia y Adicción al Smartphone (EDAS), en su versión revisada, posee un índice de validez $(V=.98)$ a partir de la evaluación de cuatro criterios de evaluación (Claridad, Contexto, Congruencia y Dominio del constructo) de 4 jueces. Respecto a la validez de contenido, se obtuvo mediante pruebas de KMO y esfericidad de Bartlett. Resultados: $(\mathrm{KMO}=.764 ; \mathrm{P}<.05)$ indica que el instrumento es apropiado para un análisis factorial confirmatorio (AFC), lo cual sugiere que el instrumento debe poseer un contenido distribuido en 3 factores que explican el $43.0 \%$ de la varianza acumulada. Respecto a la confiabilidad de instrumento este alcanzó un (.844) lo que indicaría que instrumento posee una confiabilidad adecuada. Conclusión: El instrumento evidencia una buena consistencia interna y validez de constructo.

Palabras clave: Dependencia, adicción, smartphone

\section{ABSTRACT}

Objective: To adapt and analyze the psychometric properties of the "Smartphone Dependence and Addiction" scale (EDAS) original authorship of María Aranda López, Virginia Fuentes Gutiérrez and Marta García Domingo, who created the instrument in 2017. Methodology: A sample of 117 people aged 18 to 35 years between men and women, data collection was done online through the Google form platform, the results show that the Smartphone Dependence and Addiction Scale (EDAS), revised, it has a validity index $(V=.98)$ from the evaluation of four evaluation criteria (Clarity, Context, Congruence and Construct Domain) of 4 judges. Regarding content validity, it was obtained using $\mathrm{KMO}$ and Bartlett's sphericity tests. Results: $(\mathrm{KMO}=.764 ; \mathrm{P}<.05)$ indicates that the instrument is appropriate for a confirmatory factor analysis (CFA), which suggests that the instrument must have a

${ }^{1}$ Constructora e inversiones U\&H Star S.A.C., Juliaca, Perú

Orcid ID: 0000-0002-0237-1003

${ }^{2}$ Universidad Peruana Unión, Juliaca, Perú

Orcid ID: 0000-0002-1502-7279

${ }^{3}$ Universidad Peruana Unión, Juliaca, Perú

Orcid ID: 0000-0002-7404-7464 
content distributed in 3 factors that explain $43.0 \%$ of the variance accumulated. Regarding the reliability of the instrument, this reached (.844) which would indicate that the instrument has adequate reliability. Conclusion: The instrument shows good internal consistency and construct validity.

Keywords: Dependence, addiction, smartphone

\section{INTRODUCCIÓN}

En la actualidad nuestra sociedad se rige por la utilización de herramientas tecnológicas, una de estas herramientas son los celulares inteligentes más conocidos como Smartphone los cuales son dispositivos que tienen diferentes y variadas utilidades como blog de notas, reproductor de audio y video, plataformas de juegos, programas estadísticos, programas de edición y visualización de documentos, navegadores de internet, comunicación social a través de las redes sociales: Twitter, Facebook, Instagram, Tinder, etc. El uso descontrolado y excesivo de estos dispositivos ha llevado a los expertos a plantearse la idea de que se podría generar adicción a estos aparatos, cabe recalcar que adolescentes y jóvenes son la población que los usan con mayor frecuencia estos dispositivos.

La Organización Mundial de la Salud (1982) definió la dependencia como un síndrome que incluye un patrón de comportamiento en el que existe una gran prevalencia del uso de una - varias sustancias psicoactivas, así mismo implica comportamientos con consecuencias los cuales son perjudiciales para la persona y la sociedad.

La dependencia es el estado donde se encuentran las personas que, por motivos de edad, enfermedad entre otras causas, pierden parcial o totalmente la autonomía, física, mental, intelectual o sensorial (Subdirección General de Planificación, Ordenación y Evaluación, 2011). Entonces podemos decir que la dependencia a los celulares "es la pérdida de la noción del tiempo e implica el abandono de actividades comunes, además que se pueden presentar ira, tensión y/o depresión ante la imposibilidad de acceder al celular" (Mathey, 2017).

Podemos decir que "las tecnologías no median meramente la comunicación entre sujetos, sino que además operan en los procesos por los cuales se va definiendo la identidad del sujeto en la relación con otros" (Guerra Chumbes,
2018). Siendo el caso, el problema del uso de los celulares se encuentra principalmente en el uso del entretenimiento que estos brindan comenzando por aplicaciones de redes sociales y juegos, los cuales empiezan como un pasatiempo, para luego volverse dependientes o adictos de tales actividades que les brinda el celular (Guerra Chumbes, 2018).

Una investigación realizada en España en el 2017, con el objetivo de elaborar y validar una escala para evaluar el uso excesivo y problemático de las aplicaciones implicadas a los smartphones, en dicha investigación participaron 820 personas, la mayoría fueron estudiantes de la Universidad de Jaén (España). Con edades entre 18 a 40 años $(M=22,38$, Dt $=4,53$ ), siendo un $69,3 \%$ mujeres y un $30,7 \%$ hombres. Los análisis estadísticos dieron lugar a una herramienta final constituida por 40 ítems y tres componentes (Aranda, Fuentes, \& García, 2017)

En una investigación realizada por Cuesta (2018), donde se analizaron instrumentos validados ya existentes y que miden el uso de los Smartphone, teoría, factores de estudio y propiedades psicométricas, de consistencia y de validez. Después del análisis, se concluyó que no existe una base teórica clara que apoye este como un trastorno conductual, tampoco existe un consenso sobre su inclusión en una terminología determinada, por último, utiliza criterios diagnósticos para trastornos distintos del DSM-5, un principal problema para la comparación entre escalas.

Los psicólogos indican que la adicción al celular está en aumento rápidamente, las personas adictas cada vez van en aumento y más jóvenes. Un estudio encuesto a 1000 estudiantes de corea del sur, de los cuales el $72 \%$ de los niños de 11 y 12 años tienen un móvil, usan el móvil en promedio de 5,4 horas al día, así mismo se encontró que casi el $25 \%$ de los niños son adictos al móvil (Chen, 2015). 
En la investigación realizada por Ruiz et al (2010), titulado "Análisis de comportamientos relacionados con el uso/abuso de Internet, teléfono móvil, compras y juego en estudiantes universitarios" con el objetivo de conocer los hábitos que están relacionados con las posibles conductas adictivas (juego patológico, Internet, compras, uso del teléfono móvil, etc.), que podrían presentar los jóvenes estudiantes de la Universidad de Córdoba (España), así mismo relacionar tales comportamientos con variables como la edad, sexo, curso y macro áreas de conocimiento (letras y ciencias). La investigación encontró que los estudiantes que fueron encuestados utilizan moderadamente del internet, el juego, las compras y el teléfono móvil, así mismo existe un grupo reducido de jóvenes que están cerca de tener un problema de adicción con este tipo de comportamiento (Ruiz Olivares, Lucena Jurado, Pino Osuna, \& Herruso, 2010).

En la investigación titulada "Ellas, ellos y su móvil: Uso, abuso (¿y dependencia?) del teléfono móvil en la adolescencia" realizada por Chóliz, Villanueva \& Chóliz (2009) Se realizó una encuesta con 2.486 adolescentes, entre 12 y 18 años, en la que se analizaron los principales parámetros de uso del móvil. Los objetivos principales fueron tanto la descripción del patrón de uso habitual del móvil por parte de los adolescentes, como el análisis de las diferencias de género en algunos aspectos esenciales, tales como el consumo, actitudes hacia el móvil, o funciones que se llevan a cabo, se encontró El 95\% de los participantes en el estudio indican que poseen teléfono móvil. Dicho porcentaje oscila entre el $92 \%$ de los adolescentes entre 12 y 14 años y el $99 \%$ de los adolescentes entre 17 y 18 años. Asi mismo El gasto medio de móvil es de 18 euros al mes, que oscila entre $15 €$ (grupo de edad entre 12 y 14 años) y $21 €$ en el caso de adolescentes entre 17 y 18 años. Teniendo en cuenta la modalidad de tarjeta, quienes tienen la modalidad de prepago gastan menos que los de contrato $(13,8 €$ frente a $22,8 €)$, siendo las diferencias estadísticamente significativas $(F 1,1588=119,7$; p también Los adolescentes realizan diariamente en torno a tres llamadas de voz, envían más de cuatro mensajes de texto, 10 llamadas perdidas y dedican 26,6 minutos aproximadamente al uso del móvil. El uso se incrementa los fines de semana respecto a entre semana, lo cual parece indicar que los adolescentes lo utilizan principalmente como fórmula social o de relación interpersonal. El 62,9\% de los menores lo tienen siempre conectado, incluso por la noche (Chóliz, Villanueva, \& Chóliz, 2009).

\section{METODOLOGÍA}

El presente trabajo de investigación corresponde a un tipo cuantitativo, puesto que se desea identificar el comportamiento de una variable en poblaciones con diferentes características de estudio, el diseño que posee características acordes con el diseño no experimental, es decir, no se pretende manipular una variable, esta se evaluará en su ambiente natural, además posee una clasificación transaccional, porque se recogerán datos en un solo punto de tiempo (Hernández, Fernández, y Baptista, 2014).

\section{Participantes}

La población de estudio estuvo conformada por 117 participantes de la ciudad de Puno, de ambos sexos cuyas edades van desde los 18 a 35 años. Los individuos que participaron se dedican a distintas actividades socioeconómicas entre ellas a trabajar, estudiar, realizar labores domésticas, etc.

\section{Instrumento}

El instrumento a validar fue la escala de Dependencia y Adicción al Smartphone "EDAS". Esta escala de origen español fue creada por María Aranda López, Virginia Fuentes Gutiérrez y Marta García-Domingo en el año de 2017, para identificar la existencia de una adicción a los Smartphone; la escala original está conformada por 40 ítems cuya calificación pertenece al tipo escala Likert con 5 elecciones de frecuencia. Así mismo dicho instrumento cuenta con 3 componentes los cuales son: Uso, abuso y adicción al Smartphone y sus Aplicaciones, Rasgos de Personalidad y Gasto monetario en Aplicaciones y Juegos Móviles.

\section{Procesamiento de datos}

Se recopilo la escala original como también su ficha técnica para poder empezar la adaptación a la realidad peruana. Después se contactó con psicólogos titulados y colegiados para que puedan ser jueces y se procedió a usar la técnica de validez de jueces con la formula $V$ de Aiken. Los profesionales elegidos para esta tarea 
cumplen con los requisitos indispensables que son más de 5 años de experiencia profesional, especialistas en el área de psicología clínica y forense, conocedores del marco teórico en el cual está basada esta escala. El aporte de cada juez fue de mucha importancia ya que revisaron minuciosamente cada ítem de esta escala, levantando observaciones que se tomaron en cuenta para la versión final del instrumento.

Para iniciar el análisis de datos se recogió las fichas de los 4 jueces expertos, dando como resultado algunas correcciones en los términos utilizados para nuestra sociedad. Seguidamente se recolecto las respuestas de los participantes de la plataforma de encuesta de Google y se prosiguió a vaciar los datos en el programa estadístico Excel. Para el análisis factorial e interpretación de los resultados que a continuación se muestra en las tablas pertinentes se utilizó el software estadístico SPSS versión 22.

\section{RESULTADOS}

\section{Análisis de Validez}

En la tabla 1 se puede observar la valides de cada ítem mediante la fórmula $\mathrm{V}$ de Aiken por jueces, obteniendo como resultado total de todos los ítems .98 que supera los .8 necesarios para que un instrumento sea válido. Por lo tanto, la validez de este instrumento es óptimo.

Tabla 1

Tabla de validez de jueces por $V$ de Aiken

\begin{tabular}{|c|c|c|c|c|c|}
\hline Ítem & Claridad & Congruencia & Contexto & Dom. Del constructo & Total \\
\hline 1 & 1 & 1 & 1 & 1 & 1 \\
\hline 2 & 1 & 1 & 1 & 1 & 1 \\
\hline 3 & 1 & 1 & 1 & 1 & 1 \\
\hline 4 & 1 & 1 & 1 & 1 & 1 \\
\hline 5 & 1 & 1 & 1 & 1 & 1 \\
\hline 6 & 1 & 1 & 0.8 & 1 & 0.95 \\
\hline 7 & 1 & 1 & 1 & 1 & 1 \\
\hline 8 & 1 & 1 & 1 & 1 & 1 \\
\hline 9 & 1 & 1 & 0.8 & 1 & 0.95 \\
\hline 10 & 1 & 1 & 1 & 1 & 1 \\
\hline 11 & 1 & 1 & 1 & 1 & 1 \\
\hline 12 & 1 & 1 & 1 & 1 & 1 \\
\hline 13 & 1 & 1 & 1 & 1 & 1 \\
\hline 14 & 1 & 1 & 1 & 1 & 1 \\
\hline 15 & 1 & 1 & 1 & 1 & 1 \\
\hline 16 & 1 & 1 & 1 & 1 & 1 \\
\hline 17 & 1 & 1 & 1 & 1 & 1 \\
\hline 18 & 1 & 1 & 0.8 & 1 & 0.95 \\
\hline 19 & 1 & 1 & 1 & 1 & 1 \\
\hline 20 & 0.8 & 1 & 1 & 1 & 0.95 \\
\hline 21 & 0.8 & 1 & 1 & 1 & 0.95 \\
\hline 22 & 1 & 1 & 1 & 1 & 1 \\
\hline 23 & 0.8 & 1 & 0.8 & 1 & 0.9 \\
\hline 24 & 1 & 1 & 1 & 1 & 1 \\
\hline
\end{tabular}




\section{Validez de constructo}

En la tabla 2 se puede observar los resultados para la prueba de Kayser Meyer Olkin, dando como resultado un coeficiente de, 764 , lo que indicaría que está en un valor adecuado y se puede proceder a realizar análisis factorial de la estructura del instrumento, Así mismo el coeficiente de significancia es menor a 0,05 por lo cual se corrobora lo anterior.

Tabla 2

Prueba de KMO y Bartlett

Medida Kaiser-Meyer-Olkin de adecuación de muestreo

Prueba de esfericidad de Bartlett

\begin{tabular}{lr} 
Aprox. Chi-cuadrado & 997,033 \\
gl & 276 \\
Sig. &, 000 \\
\hline
\end{tabular}

En la tabla 3 se puede observar que el valor acumulado de la varianza total explicada es de $43.0 \%$ lo cual contrasta con la matriz del instrumento y guarda relación con su estructura que la divide en tres dimensiones: Uso, abuso y adicción a los Smartphone y sus aplicaciones, Rasgos de personalidad y Gasto monetario en aplicaciones y juegos móviles.

Tabla 3

Varianza total explicada

\begin{tabular}{|c|c|c|c|c|c|c|}
\hline \multirow{2}{*}{ Componente } & \multicolumn{3}{|c|}{ Auto valores iniciales } & \multicolumn{3}{|c|}{ Sumas de extracción de cargas al cuadrado } \\
\hline & Total & $\%$ de varianza & $\%$ acumulado & Total & $\%$ de varianza & $\%$ acumulado \\
\hline 1 & 5,956 & 24,817 & 24,817 & 5,217 & 21,736 & 21,736 \\
\hline 2 & 2,367 & 9,863 & 34,681 & 2,777 & 11,569 & 33,306 \\
\hline 3 & 2,020 & 8,417 & 43,098 & 2,350 & 9,792 & 43,098 \\
\hline 4 & 1,482 & 6,176 & 49,274 & & & \\
\hline 5 & 1,313 & 5,470 & 54,744 & & & \\
\hline 6 & 1,158 & 4,824 & 59,568 & & & \\
\hline 7 & 1,036 & 4,318 & 63,886 & & & \\
\hline 8 & ,986 & 4,110 & 67,996 & & & \\
\hline 9 & ,892 & 3,716 & 71,711 & & & \\
\hline 10 & ,792 & 3,300 & 75,011 & & & \\
\hline 11 & ,708 & 2,950 & 77,962 & & & \\
\hline 12 & ,637 & 2,655 & 80,616 & & & \\
\hline 13 &, 598 & 2,492 & 83,108 & & & \\
\hline 14 &, 566 & 2,356 & 85,465 & & & \\
\hline 15 &, 540 & 2,250 & 87,714 & & & \\
\hline 16 & ,491 & 2,047 & 89,761 & & & \\
\hline 17 & ,450 & 1,876 & 91,638 & & & \\
\hline 18 & ,421 & 1,755 & 93,393 & & & \\
\hline 19 & ,369 & 1,539 & 94,932 & & & \\
\hline 20 & ,334 & 1,390 & 96,322 & & & \\
\hline 21 & ,309 & 1,286 & 97,608 & & & \\
\hline 22 & ,223 & ,931 & 98,539 & & & \\
\hline 23 & ,200 & ,833 & 99,371 & & & \\
\hline 24 & ,151 & ,629 & 100,000 & & & \\
\hline
\end{tabular}

Método de extracción: análisis de componentes principales. 
En la tabla 4 se puede observar los valores de las cargas factoriales de cada ítem los cuales están distribuidos tres componentes: los ítems 1,2,3,4,5,6,9,10,11,13,15,16,17,21,23,24 pertenecerían a la dimensión Uso, abuso y adicción a los Smartphone y sus aplicaciones, el ítem 7,8,12,19,22 configuran en la dimensión de Rasgos de personalidad, y finalmente los ítems 14,18,20 configuran a la dimensión de Gasto monetario en aplicaciones y juegos móviles. Así ningún ítem carece de carga factorial y todos son mayores a ,3 por lo tanto ningún ítem debe ser eliminado.

Tabla 4

Estructura Factorial de la escala

\begin{tabular}{|c|c|c|c|}
\hline & $\begin{array}{l}\text { Uso, abuso y adicción a } \\
\text { los Smarphone y sus apli- } \\
\text { caciones }\end{array}$ & Rasgos de personalidad & $\begin{array}{l}\text { Gasto monetario en aplica- } \\
\text { ciones y juegos móviles }\end{array}$ \\
\hline Item 1 &, 676 & & \\
\hline Item 13 & ,667 & & \\
\hline Item 5 & ,631 & & \\
\hline Item 4 &, 597 & & \\
\hline Item 9 &, 587 & & \\
\hline Item 11 &, 571 & & \\
\hline Item 17 &, 554 & & \\
\hline Item 23 &, 552 & & \\
\hline Item 2 &, 538 & & \\
\hline Item 10 &, 537 & & \\
\hline Item 3 &, 507 & & \\
\hline Item 21 &, 505 & & \\
\hline Item 6 & ,497 & & \\
\hline Item 15 & ,467 & & \\
\hline Item 24 & ,405 & & \\
\hline Item 16 & ,384 & & \\
\hline Item 22 & & ,778 & \\
\hline Item 12 & & ,643 & \\
\hline Item 19 & & ,628 & \\
\hline Item 8 & & ,627 & \\
\hline Item 7 & & ,457 & \\
\hline Item 20 & & & ,913 \\
\hline Item 18 & & & ,889 \\
\hline Item 14 & & & ,445 \\
\hline
\end{tabular}

Método de extracción: análisis de componentes principales.

\section{Confiabilidad}

En la tabla 5 se puede observar el coeficiente de Alfa de Cronbach que es .844, para las propiedades psicométricas de la Escala de Dependencia y Adicción al Smartphone "EDAS" superando el valor mínimo permitido, por lo tanto, el nivel de confiabilidad del instrumento es adecuado. 
Tabla 5

Estadísticos de confiabilidad por Alfa de Cronbach

\begin{tabular}{ll} 
Alfa de Cronbach & N de elementos \\
.844 & 24 \\
\hline
\end{tabular}

En la tabla 6 se puede observar los estadísticos totales por elemento, así como la confiabilidad de cada ítem.

Tabla 6

Estadísticos totales del elemento

\begin{tabular}{|c|c|c|c|c|}
\hline & $\begin{array}{l}\text { Media de escala si el } \\
\text { elemento se ha supri- } \\
\text { mido }\end{array}$ & $\begin{array}{l}\text { Varianza de escala si el } \\
\text { elemento se ha supri- } \\
\text { mido }\end{array}$ & $\begin{array}{l}\text { Correlación total de } \\
\text { elementos corre- } \\
\text { gida }\end{array}$ & $\begin{array}{l}\text { Alfa de Cronbach si } \\
\text { el elemento se ha } \\
\text { suprimido }\end{array}$ \\
\hline Item 1 & 40,67 & 110,776 &, 557 & 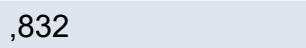 \\
\hline Item 2 & 40,24 & 111,977 &, 572 & ,833 \\
\hline Item 3 & 40,77 & 113,386 & ,385 & ,839 \\
\hline Item 4 & 40,36 & 111,405 &, 576 & ,832 \\
\hline Item 5 & 40,85 & 114,125 & ,332 & ,841 \\
\hline Item 6 & 40,56 & 107,766 &, 529 & ,832 \\
\hline Item 7 & 40,45 & 111,388 & ,479 & ,835 \\
\hline Item 8 & 39,69 & 121,560 & ,023 & ,851 \\
\hline Item 9 & 41,00 & 112,948 & ,431 & ,837 \\
\hline Item 10 & 40,53 & 112,700 & ,404 & ,838 \\
\hline Item 11 & 41,06 & 114,091 & ,391 & ,838 \\
\hline Item 12 & 39,93 & 113,513 & ,447 & ,837 \\
\hline Item 13 & 40,75 & 108,188 & ,621 & ,829 \\
\hline Item 14 & 41,25 & 113,412 & ,308 & ,843 \\
\hline Item 15 & 40,79 & 113,940 & ,357 & ,840 \\
\hline Item 16 & 40,67 & 113,983 & ,362 & ,839 \\
\hline Item 17 & 40,61 & 112,085 &, 518 & ,834 \\
\hline Item 18 & 41,93 & 117,668 & ,239 & ,843 \\
\hline Item 19 & 40,05 & 114,463 & ,435 & ,837 \\
\hline Item 20 & 41,96 & 118,472 & ,170 & ,846 \\
\hline Item 21 & 40,18 & 111,580 & ,551 & ,833 \\
\hline Item 22 & 39,72 & 121,549 & ,015 & ,852 \\
\hline Item 23 & 40,95 & 111,532 & ,437 & ,837 \\
\hline Item 24 & 40,86 & 113,688 & ,398 & ,838 \\
\hline
\end{tabular}

\section{Baremación adaptada}

Se elaboró la baremación de este instrumento a partir de su aplicación en 117 sujetos, distribuidos en 4 cortes donde según los percentiles convertidos a puntuaciones dan los siguientes resultados de 0-36, el encuestado no presenta conductas de dependencia y adicción; si el puntaje es de 37-41, el encuestado tiene alto riesgo de presentar conductas de dependencia y adicción; un puntaje entre 
42-48 significa que el encuestado presenta conductas de dependencia y adicción; y una puntuación de 49 a más significa que el encuestado presenta elevadas conductas de dependencia y adicción al Smartphone y sus aplicaciones. Cuanto más alto es el puntaje, mayor será el grado de conductas de dependencia y adicción.

Tabla 7

Tabla Percentilar con valores categóricos en 4 cortes

\begin{tabular}{|c|c|c|c|c|c|}
\hline $\mathrm{Pc}$ & $\begin{array}{l}\text { Uso, abuso y } \\
\text { adicción a los } \\
\text { Smartphone y sus } \\
\text { aplicaciones }\end{array}$ & $\begin{array}{l}\text { Rasgos de per- } \\
\text { sonalidad }\end{array}$ & $\begin{array}{l}\text { Gasto monetario } \\
\text { en aplicaciones y } \\
\text { juegos móviles }\end{array}$ & Total & $\begin{array}{l}\text { Nivel de dependencia y } \\
\text { adicción }\end{array}$ \\
\hline $5-25$ & $0-27$ & $0-6$ & $0-1$ & $0-36$ & $\begin{array}{l}\text { No presenta conductas } \\
\text { de dependencia y adic- } \\
\text { ción }\end{array}$ \\
\hline $30-45$ & $28-31$ & 7-8 & $2-5$ & $37-41$ & $\begin{array}{l}\text { Alto riesgo de presentar } \\
\text { conductas de dependen- } \\
\text { cia y adicción }\end{array}$ \\
\hline $50-70$ & $32-36$ & $9-13$ & $6-10$ & $42-48$ & $\begin{array}{l}\text { Presenta conductas de } \\
\text { dependencia y adicción }\end{array}$ \\
\hline 75-99 & 37 a más & 14 a más & 11.12 & $\begin{array}{l}49 \text { a } \\
\text { más }\end{array}$ & $\begin{array}{l}\text { Elevado Nivel conductas } \\
\text { de dependencia y adic- } \\
\text { ción }\end{array}$ \\
\hline
\end{tabular}

Tabla 8

ítems del EDAS

N Preguntes

1 Siento ansiedad si me quedo sin batería en el celular

2 Miro alguna de las aplicaciones del celular (por ejemplo, WhatsApp, Instagram, Facebook, etc.) cada 5 minutos aproximadamente.

3 Mi familia, pareja, amigos, se han quejado alguna vez del tiempo que ocupo mirando el celular.

4 Dedico un tiempo excesivo a mirar distintas aplicaciones del celular (Facebook, WhatsApp, juegos, etc.)

5 El concepto que tengo que de mí mismo/a depende de cómo me ven los demás.

6 Regresaría a casa por mi celular si me doy cuenta de que lo olvidé, aunque ya esté alejado de ella.

$7 \quad$ Miro frecuentemente el celular en clase o en el trabajo. 


N Preguntas Nunca $\begin{aligned} & \text { Casi } \\ & \text { nunca }\end{aligned} \quad$ A veces $\begin{aligned} & \text { Casi } \\ & \text { siempre }\end{aligned}$ Siempre

8 Mi autoestima es elevada.

9 Mi principal motivación para e salir en una fotografía es compartirla inmediatamente a través de cualquier medio del celular.

10 Suelo agotar la batería antes de que acabe el día.

11 El uso del celular ha interferido alguna vez con mi desempeño académico y/o laboral.

12 Lo primero que hago al levantarme es mirar el celular.

13 Me siento mal si no miro inmediatamente el celular si recibo las señales de alerta ante un mensaje (sonido, vibración, luz o icono)

14 Realizo pagos periódicos por aplicaciones.

15 He intentado disminuir el uso que hago del celular, pero no puedo.

Mi comportamiento es más desinhibido cuando

16 uso las aplicaciones de mensajería y redes sociales del celular que en mi vida diaria.

Suelo utilizar el celular cuando estoy en situacio-

17 nes sociales con varias personas (en un bar con los amigos, en una comida familiar, etc.)

18 Utilizo juegos en el celular que me cuestan dinero.

19 Soy una persona extrovertida.

20 He gastado dinero en comprar aplicaciones para el celular.

21 Me relaja utilizar el celular.

22 Estoy a gusto con mi físico y mi manera de ser.

Alguna vez he considerado que tengo un problema

23 con alguna de las aplicaciones del celular por el tiempo o coste que me supone.

24 Cuando no estoy localizable me preocupo con la idea de perderme alguna llamada. 


\section{DISCUSIÓN}

La adicción a los Smartphone o teléfonos inteligentes por su traducción en inglés es un reciente pero amplio objetivo de estudio en los últimos años, ya que debido a frecuentes escenas donde más personas reaccionan de manera negativa sobre su conducta cuando les quitan el celular, es por ello la preocupación de los profesionales de la salud mental entre ellos psicólogos y psiquiatras ,a pesar de que la única adicción comportamental categorizada como tal en el DSM-5 (American Psychiatric Association, 2013) es la adicción al juego de azar, se ha encontrado evidencia de la similitud la adicción al uso del celular y el resto de adicciones,

El objetivo de este estudio fue de analizar las propiedades psicométricas de la Escala EDAS, a través del análisis factorial exploratorio, en una muestra de individuos cuyas edades fueron de 18 a 35 años, ya que en nuestra región son las personas con un mayor uso de frecuencia de los Smartphone por factores económicos y sociales.

El análisis factorial exploratorio realizado, indica que la nueva versión adaptada mantiene relación con la estructura interna del instrumento en su versión original, ya que los autores originales elaboraron dicha escala con tres factores.

La validación de la escala EDAS realizada en colaboradores voluntarios obtuvo aceptables valores de consistencia interna, un $\mathrm{V}$ de Aiken $=.98$. Además, se realizó el análisis factorial exploratorio por KMO obteniendo el resultado de .764 lo que significaría que el nivel de significancia es óptimo dentro de un análisis factorial exploratorio y confirmatorio para proseguir con el análisis de confiabilidad de la escala, después del análisis respectivo de obtuvo como coeficiente .844 lo que significa que el instrumento tiene una confiabilidad adecuada.

Comparando con otros estudios hallados, Guerra (2018) realizó una investigación con la finalidad de construir una Escala de adicción a las redes sociales para estudiantes de secundaria del distrito de Carmen de La Legua Reynoso, 2018, mediante un estudio de diseño instrumental y de tipo psicométrico y tecnológico, no experimental con la participación de 1,202 estudiantes de primero a quinto grado de secundaria de ambos sexos, obteniendo como resultado que la Escala de adicción a las redes sociales tiene un grado adecuado de confiabilidad de 788 , el cual se obtuvo a través del Alfa de Cronbach. El cual es un valor menor que este estudio teniendo en cuanta que la población es ampliamente mayor en la presente comparación.

Así mismo Ávila (2016) realizó una investigación con la finalidad de estudiar las características psicométricas de la Escala de adicción al internet de Lima - EAIL en estudiantes de una Institución distrital de Bogotá, mediante un estudio de tipo no experimental con corte descriptivo con una muestra de 110 participantes en edades de 11 y 18 años, obteniendo como resultado que la Escala de adicción al internet de Lima - EAIL tiene un grado a de confiabilidad aceptable del instrumento de 79 , el cual se obtuvo a través del Alfa de Cronbach. El cual es un valor inferior al del presente estudio teniendo en cuenta que la población de estudio es similar en ambas investigaciones.

Por lo contrario, Vite (2018) realizo una investigacióntitulada"Adaptacióndelcuestionario de adicción a las redes sociales en estudiantes de tercero a quinto año de secundaria de cuatro colegios públicos-Independencia, Lima, 2018" teniendo como población de estudios 803 estudiantes en edades de 13 a 17 años, dando como resultado una confiabilidad adecuada del instrumento de ,92 Alfa de Cronbach. El cual es superior al de la investigación teniendo en cuenta que la población es mucho mayor en dicha investigación. Además, Cabos y Guarniz (2020) realizaron una investigación que tuvo como objetivo principal determinar las evidencias de validez y confiabilidad del cuestionario de adicción a redes sociales en ingresantes a una universidad trujillana como población de estudio 498 participantes entre 19 y 25 años de edad, dando como resultado una confiabilidad adecuada del instrumento de ,94 Alfa de Cronbach. El cual es superior al de la investigación teniendo en cuenta que la población es mucho mayor en dicha investigación.

Es por ello que la utilización de Escala de Dependencia y Adicción al Smartphone "EDAS" para medir conductas adictivas al Smartphone es total mente viable y recomendable para el uso profesional ya que a comparación de otros estudios similares tiene un adecuado nivel de confiabilidad y al ser un instrumento de magnitud completa para el enfoque actual ya que no solo mide niveles de dependencia 
y adicción a las redes sociales como se ha apreciado en las otras investigaciones, sino que mide niveles de dependencia y adicción en todas las herramientas informáticas que ofrece el Smartphone y al ser un aparato tecnológico con mayor frecuencia de uso se convierte en un vector principal para el acceso a la tecnología actual.

La limitación del presente estudio reside en que por la situación actual de confinamiento y recorte de actividades diarias en la mayoría de las personas y la población en estudio se ha elevado el uso de los Smartphone en niveles casi indiscriminado, es por eso que se debería realizar un estudio donde se utilice este instrumento y haga el análisis correlativo pre y pos pandemia.

\section{Declaración de financiamiento y de conflictos de interés:}

El estudio fue financiado por los autores, quienes declaran no tener algún tipo de conflicto de interés en la investigación realizada.

\section{Correspondencia}

Aurora Lucana Hancco

Correo electrónico:

aurora.lucana@upeu.edu.pe

Heber Pari Betancur

Correo electrónico:

heber.pari@upeu.edu.pe

Alcides Quispe Mamani

Correo electrónico:

alcides.quispe@upeu.edu.pe

\section{REFERENCIAS BIBLIOGRÁFICAS}

Ávila Berrio, D., Pardo Jaime, E., \& Muñoz Rojas, M. (2019). Escala de Adicción al Internet de Lima (EAIL): Análisis psicométrico. Revista Iberoamericana De Psicología, 11(3), 103-111. https://doi.org/10.33881/2027-1786.rip.11309
Aranda, M., Fuentes, V., \& García, M. (2017). "No sin mi Smartphone": Elaboración y validación de la Escala de Dependencia y Adicción al Smartphone (EDAS). Terapia Psicológica, 35(1), 35-45.

Cabos, E., Guarniz, W., Cruz, H., \& Guerrero, V. (2020). Evidencias de Validez y Confiabilidad del Cuestionario de Adicción a Redes Sociales en Ingresantes a una Universidad de Trujillo. Universidad César Vallejo. Retrieved from https://repositorio.ucv.edu.pe/bitstream/ handle/20.500.12692/43465/Cabos_SEGGuarniz_RWNAK.pdf?sequence=1\&isAllowed=y

Chen, H. (2015). El miedo a quedarse sin celular: un trastorno que llegó para quedarse - BBC News Mundo. BBC. Retrieved from https:// www.bbc.com/mundo/noticias/2015/09/150907 tecnologia_nomofobia_ansiedad_moviles_men

Chóliz, M., Villanueva, V., \& Chóliz, M. C. (2009). Ellas, ellos y su móvil: Uso, abuso (¿y dependencia?) del teléfono móvil en la adolescencia. Revista Española de Drogodependencias Originales, 34(1), 74-88.

Guerra, E., De la Cruz, C., \& Olivas, L. (2018). Construcción de una Escala de adicción a las redes sociales para estudiantes de secundaria del distrito de Carmen de La Legua Reynoso, 2018. Universidad César Vallejo.

Guerra, J. (2018). Uso del celular y su relación con el aprendizaje en los estudiantes de la Institución Educativa Publica "Víctor Manuel Maurtua" en Parcona Ica. Universidad Nacional de Huancavelica. Retrieved from https://1library.co/ document/yngldgkz-celular-relacion-aprendizajeestudiantes-institucion-educativa-publica-victor. html

Ruiz-Olivares, R., Lucena, V., Pino, M. J., \& Herruzo, J. (2010). Análisis de comportamientos relacionados con el uso/abuso de Internet, teléfono móvil, compras y juego en estudiantes universitarios. Adicciones, 22(4), 301. https://doi. org/10.20882/adicciones.171
Recibido: 10/02/2021

Aceptado: 20/05/2021 